ARTICLE

\title{
An ankyrin-repeat and WRKY-domain-containing immune receptor confers stripe rust resistance in wheat
}

\author{
Huan Wang (1,2, Shenghao Zou', Yiwen Li id ${ }^{2}$, Fanyun Lin (D) $^{2} \&$ Dingzhong Tang ${ }^{1 凶}$
}

Perception of pathogenic effectors in plants often relies on nucleotide-binding domain (NBS) and leucine-rich-repeat-containing (NLR) proteins. Some NLRs contain additional domains that function as integrated decoys for pathogen effector targets and activation of immune signalling. Wheat stripe rust is one of the most devastating diseases of crop plants. Here, we report the cloning of $\mathrm{YrU1}$, a stripe rust resistance gene from the diploid wheat Triticum urartu, the progenitor of the A genome of hexaploid wheat. YrU1 encodes a coiled-coil-NBS-leucinerich repeat protein with $\mathrm{N}$-terminal ankyrin-repeat and C-terminal WRKY domains, representing a unique NLR structure in plants. Database searches identify similar architecture only in wheat relatives. Transient expression of YrU1 in Nicotiana benthamiana does not induce cell death in the absence of pathogens. The ankyrin-repeat and coiled-coil domains of YrU1 selfassociate, suggesting that homodimerisation is critical for YrU1 function. The identification and cloning of this disease resistance gene sheds light on NLR protein function and may facilitate breeding to control the devastating wheat stripe rust disease.

\footnotetext{
${ }^{1}$ State Key Laboratory of Ecological Control of Fujian-Taiwan Crop Pests, Key Laboratory of Ministry of Education for Genetics, Breeding and Multiple Utilization of Crops, Plant Immunity Center, Fujian Agriculture and Forestry University, Fuzhou 350002, China. ${ }^{2}$ State Key Laboratory of Plant Cell and Chromosome

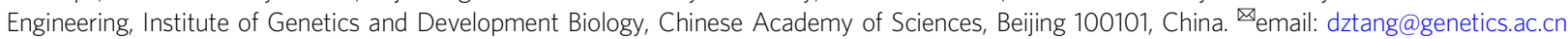


W heat stripe rust, caused by Puccinia striiformis f. sp. tritici (Pst), is one of the most devastating diseases of crops, causing major production losses in hexaploid wheat (Triticum aestivum L.) around the world ${ }^{1}$. Stripe rust has become the most damaging of all the crop rusts due to its expanding range and the resulting increased production losses ${ }^{2}$. To date, over 80 stripe rust resistance genes have been identified and mapped in wheat $^{3}$, but only a few, including $Y r 5, Y r 7, Y r S P$, Yr15, Yr18/Lr34, Yr36, Yr46 and YrAS2388, have been cloned ${ }^{4-9}$. Among these, $Y r 18 / L r 34$ encodes a putative ATP-binding cassette (ABC) transporter; Yr36 encodes a protein with a kinase domain and a putative lipid-binding domain; Yr46 encodes a hexose transporter; and Yr15 encodes a protein with predicted kinase and pseudokinase domains. These genes encode different protein families and confer a relatively broad spectrum of resistance. In contrast, YrAS2388 encodes a nucleotide-binding site (NBS) and leucine-rich repeat (LRR) proteins (NLRs), and $Y r 5, Y r 7$ and YrSP encode NLR with additional BED domain; all of these genes are race specific, being effective against only a subset of Pst isolates at the seedling or all stages ${ }^{4-9}$. The robustness of stripe rust resistance genes has been challenged by coevolution between wheat and stripe rust pathogens in which the pathogens have evolved the capacity to overcome the resistance conveyed by these genes $^{2,10}$. For instance, $\operatorname{Yr} 7$, which confers resistance to many stripe rust isolates, has been overcome by newer isolates ${ }^{11,12}$.

Plants use intracellular NLRs to detect pathogens by direct or indirect recognition of pathogen effectors ${ }^{13}$. Most NLRs contain three domains: an N-terminal variable domain, NBS domain and C-terminal $\mathrm{LRR}^{13,14}$. Several also have additional functional domains, which are hypothesised to act as integrated decoys. For instance, the Arabidopsis thaliana (Arabidopsis) resistance protein RRS1 has a C-terminal WRKY domain; the rice (Oryza sativa) resistance protein RGA5 has a C-terminal RATX1 domain; the wheat stripe rust resistance proteins Yr5, Yr7 and YrSP have an N-terminal BED domain; and the tomato resistance proteins $\mathrm{Sw}-5 \mathrm{~b}$ and Prf have an extra N-terminal domain ${ }^{7,15-18}$. Among these, the RATX1 domain of RGA5 directly binds to a Magnaporthe oryzae effector, and activates RGA4-mediated resistance and cell death upon recognition ${ }^{17,19}$. The WRKY domain of RRS1 functions as a decoy to bind the pathogen effector and triggers immune signalling mediated by its neighbouring NLR RPS4 ${ }^{20,21}$. The additional domains in other NLRs are not well characterised.

The diploid wheat species T. urartu is the progenitor species of the A genome of common wheat and a potential source for stripe rust resistance genes ${ }^{22,23}$. The high-quality genome sequence of T. urartu has been published and can be used for map-based cloning of genes ${ }^{24,25}$. In this study, we map-based cloned a stripe rust resistance gene from the resistant $T$. urartu accession PI428309. The gene encodes a CC-NBS-LRR protein with an Nterminal ankyrin-repeat (ANK) domain and a C-terminal WRKY domain. The ANK-NLR-WRKY domain is rare, and this type of protein/gene structure is found only in the genomes of wheat and its relatives. The identification and cloning of this type of stripe rust resistance gene will not only help to uncover new mechanism of disease resistance but also facilitate breeding for disease resistance in wheat.

\section{Results}

Triticum urartu PI428309 is resistant to stripe rust. To identify stripe rust resistance genes in $T$. urartu, we infected a large number of $T$. urartu accessions at the seedling stage with the Puccinia striiformis f. sp. tritici (Pst) isolate CYR33 and observed the disease severity 14 days later. G1812, the accession used for the reference genome sequence ${ }^{24}$, was highly susceptible to Pst
CYR33, displaying a large number of visible uredia (spore-bearing pustules characteristic of rust disease) and lacked visible cell death (which is indicative of a resistance response) on the leaves at 14 days post inoculation (dpi; Fig. 1a, Supplementary Fig. 1). However, several T. urartu accessions showed resistance to Pst CYR33, with very few or no uredia and prominent cell death 14 dpi (Supplementary Fig. 1).

We selected for further characterisation the accession PI428309, which showed resistance to five stripe rust races, CYR17, CYR31, CYR32, CYR33, and V26 (Fig. 1a, Supplementary Fig. 2), and was recently used to clone a powdery mildew resistance gene, $P m 60^{26}$. At $2 \mathrm{dpi}$, after wheat germ agglutinin (WGA) staining, fluorescence micrographs of the fungal structures showed that substomatal vesicles (SSV), primary infection hyphae (PH) and haustoria mother cells (HMCs) were formed in both PI428309 and the susceptible accession G1812, and haustoria were already found in G1812, but not in PI428309 (Fig. 1b). At 7 dpi, fewer fungal colonies and pathogen feeding structures were observed in PI428309 than in G1812 (Fig. 1c). We examined the transcript levels of several pathogenesis-related genes and found that, consistent with the resistant phenotype in PI428309, the pathogenesis-related genes $P R 1, P R 2, P R 3$, and PR5 were induced to much higher levels in PI428309 than in G1812 (Supplementary Fig. 3), indicating that resistance responses were strongly activated in PI428309.

To study the genetic basis of stripe rust resistance in PI428309, we crossed PI428309 and G1812 and evaluated the disease resistance response of the $\mathrm{F}_{1}$ plants when infected with Pst CYR33. The $\mathrm{F}_{1}$ plants were resistant to CYR33, producing limited uredia and showing prominent cell death on the leaves at $14 \mathrm{dpi}$ (Fig. 1); their infection types were similar but slightly higher than those of the PI428309 plants. Next, we inoculated the $F_{2}$ plants with Pst CYR33 and phenotyped individual plants for stripe rust resistance. The segregation ratio of disease resistance in the $F_{2}$ generation showed that the resistance to Pst CYR33 in PI428309 was controlled by one major dominant resistance locus, which we tentatively named $\operatorname{Yr}$ (Supplementary Table 1).

Genetic and physical maps of the resistance gene. To map the Yr stripe rust resistance gene in PI428309, we screened 259 simple sequence repeat (SSR) markers on the common wheat chromosomes (1A to 7A) to analyse the polymorphisms between PI428309 and G1812. Eighty-two polymorphic markers were used to screen the susceptible plants of the $F_{2}$ population derived from the cross between PI428309 and G1812. We discovered that marker Xgpw7007-5A (Fig. 2a), in the wheat deletion-line bin 5AL10-0.57-0.78 of the T. aestivum cultivar Chinese Spring, was linked with the stripe rust resistance locus.

To identify more molecular markers tightly linked to the stripe rust resistance locus, we used the wheat expressed sequence tags (ESTs) in the deletion bins 5AL12-0.35-0.57 and 5AL10-0.57-0.78 to search the genome sequences of G1812 using BLASTN. We then used the scaffolds of G1812 in the deletion-line bins to develop SSR markers. Through this method, we developed several markers closely linked with the stripe rust resistance locus $Y r$, including SCF12 (from scaffold 68558), SCF13 (from scaffold 21752), SCF19 (from scaffold 9979) and SCF20 (from scaffold 49434) (Fig. 2a, Supplementary Table 2). To further identify new markers, we used the Brachypodium distachyon genes collinear to $Y r$ to search the genome sequences of G1812 using BLASTN, and then develop additional SSR and cleaved amplified polymorphic sequence (CAPS) markers with those sequences (Supplementary Table 3). Using 3,034 susceptible plants, identified among a total of $11,906 \mathrm{~F}_{2}$ plants from the cross between PI428309 and G1812, we ultimately anchored the $Y r$ resistance locus to a region of 
a
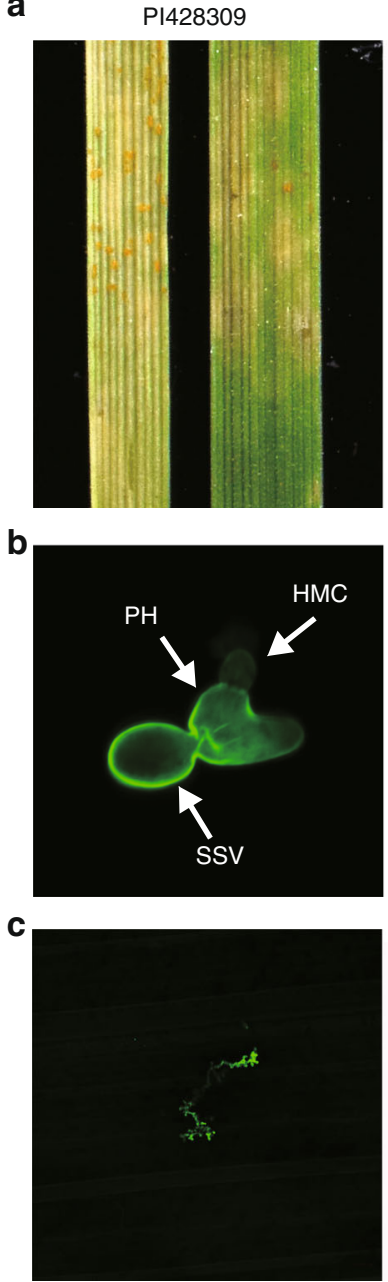

$\mathrm{PI} 428309 / \mathrm{G} 1812 \mathrm{~F}_{1}$
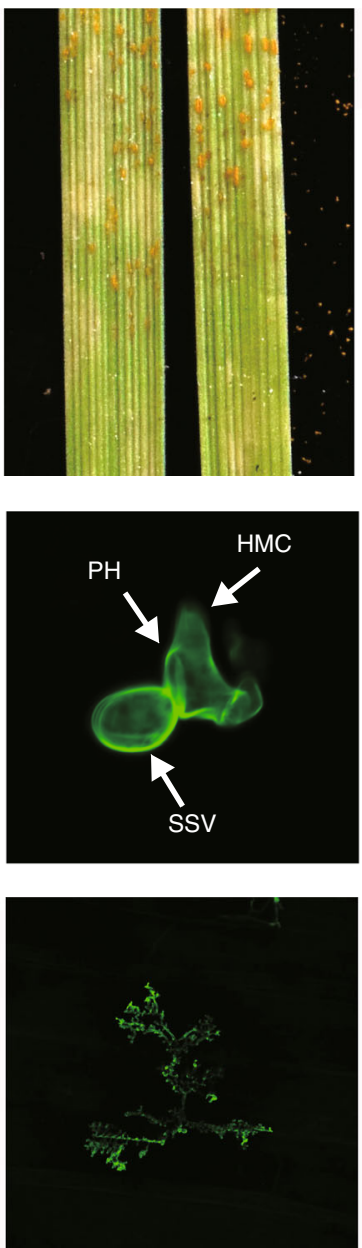

G1812
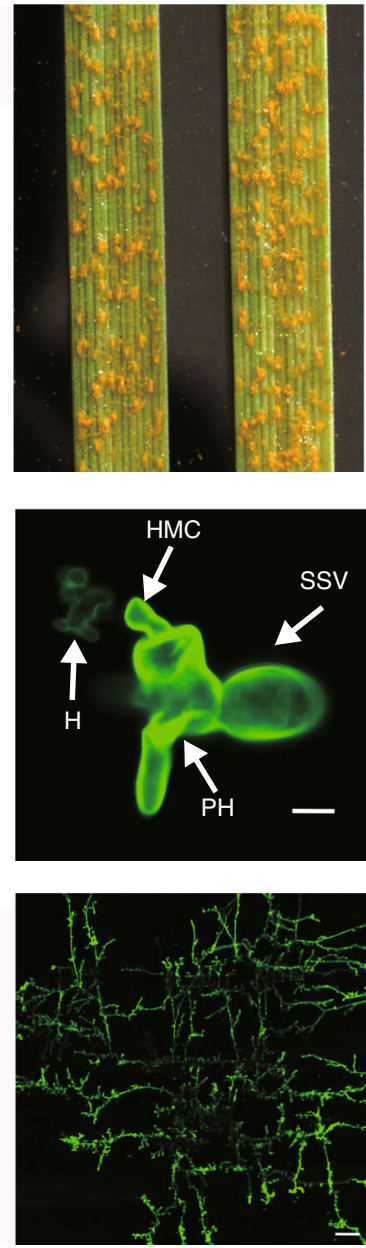

Fig. 1 T. urartu PI428309 showed resistance to stripe rust pathogen Puccinia striiformis f. sp. tritici (Pst) CYR33. a Ten-day-old plants of accessions PI428309 and G1812 and F progeny of a cross between PI428309 and G1812 were infected with Pst CYR33. Plant leaves were detached and photographed at 14 dpi. PI428309 (infection type (IT) 1) and $F_{1}$ (IT 2) showed resistance to Pst CYR33, while G1812 (IT 4) showed susceptibility to Pst CYR33. b, c Fluorescence micrographs of the fungal structures of Pst CYR33 at 2 and 7 dpi in PI428309, G1812 and $F_{1}$ plants. The fungal structures were stained with wheat germ agglutinin (WGA). SSV, substomatal vesicle; PH, primary infection hyphae; HMC, haustoria mother cells; $\mathrm{H}$, haustoria. Bars, $10 \mu \mathrm{m}$ (b), $100 \mu \mathrm{m}$ (c).

$65 \mathrm{~kb}$ in the G1812 chromosome 5AL (Fig. 2c). This region contains three candidate genes, TuG1812G0500003718, TuG1812G0500003719 and TuG1812G0500003720, in G1812.

Cloning of the stripe rust resistance gene in PI428309. TuG1812G0500003718, TuG1812G0500003719 and TuG1812G05 00003720 all encode NLRs. We obtained two candidate genes in PI428309 allelic to TuG1812G0500003718 and TuG1812G05000 03719, which we designated $\mathrm{YrU1}$ and CG2 based on subsequent analysis (Supplementary Data 1). There is no allelic gene of TuG1812G0500003720 in PI428309 because of sequence differences between PI428309 and G1812. We analysed the sequences of YrU1 and CG2 in PI428309 and G1812 and found that YrU1 has $\sim 1 \mathrm{~Kb}$ of additional sequence in PI428309 as compared with G1812 (Supplementary Fig. 4). There were no significant sequence differences in CG2 between PI428309 and G1812 except for a few single-nucleotide polymorphisms (SNPs, Supplementary Fig. 5). The additional $\sim 1 \mathrm{~Kb}$ in PI428309 YrU1 encode a WRKY domain that is absent in G1812 (Supplementary Figs. 4 and 6). We designed a marker based on this $1 \mathrm{~Kb}$ sequence to screen for its presence in different $T$. urartu accessions and found that this sequence was present only in resistant accessions, but not in any susceptible accessions we examined (Supplementary Fig. 4). Among 157T. urartu accessions investigated, those containing the additional sequence detected by this marker were all resistant to stripe rust Pst CYR33 (Supplementary Table 4).

We also examined the transcript levels of $\mathrm{YrU} 1$ and CG2 in PI428309 and G1812 before and after inoculation with Pst CYR33 by quantitative reverse transcription PCR (qRT-PCR). The expression of $\mathrm{YrU1}$ was only slightly affected by infection (Supplementary Fig. 7), but the expression of CG2 was not detected either before or after inoculation in either G1812 or PI428309. Based on these results, we selected $\mathrm{YrU1}$ for further investigation as the candidate gene.

YrU1 knockdown suppresses PI428309 resistance to Pst CYR33. To determine whether $Y r U 1$ is responsible for resistance to stripe rust Pst CYR33 in PI428309, we transiently silenced YrU1 in T. urartu PI428309 using the Barley stripe mosaic virus (BSMV)-induced gene silencing system. This silencing impaired the plants' resistance to stripe rust Pst CYR33. At $14 \mathrm{dpi}$, the leaf surfaces of BSMV:YrU1-treated plants displayed numerous visible uredia, in contrast with the BSMV:GFP control plants, which had 
a

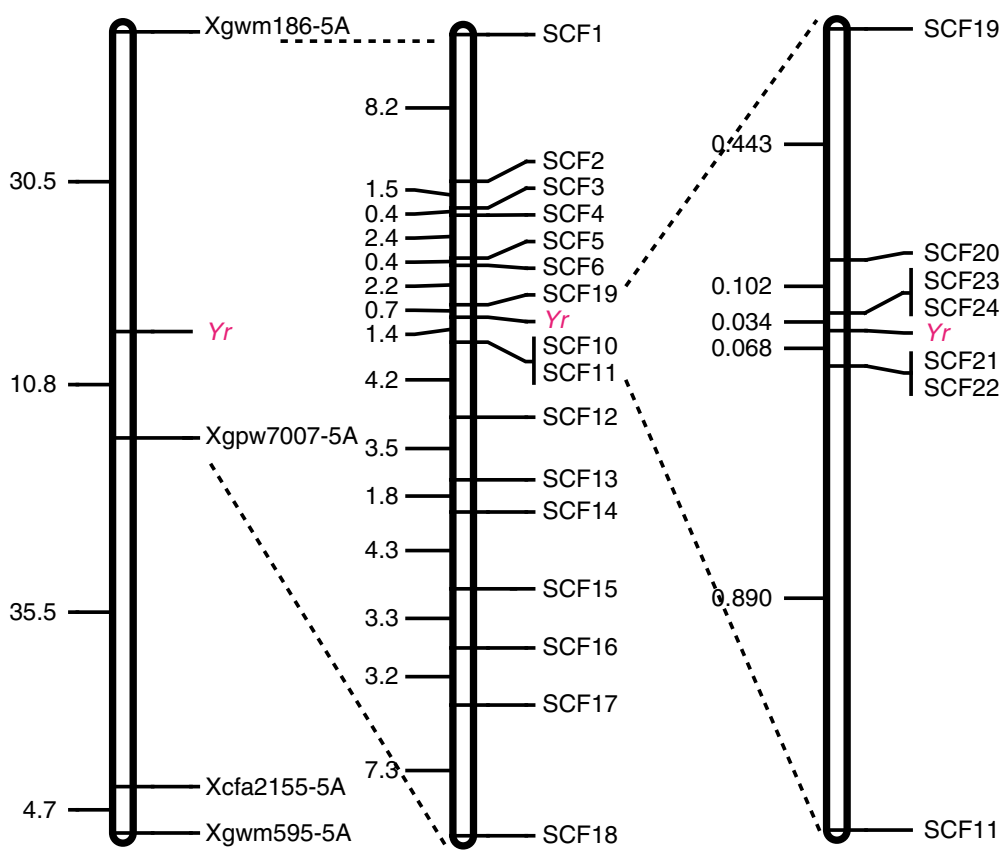

b

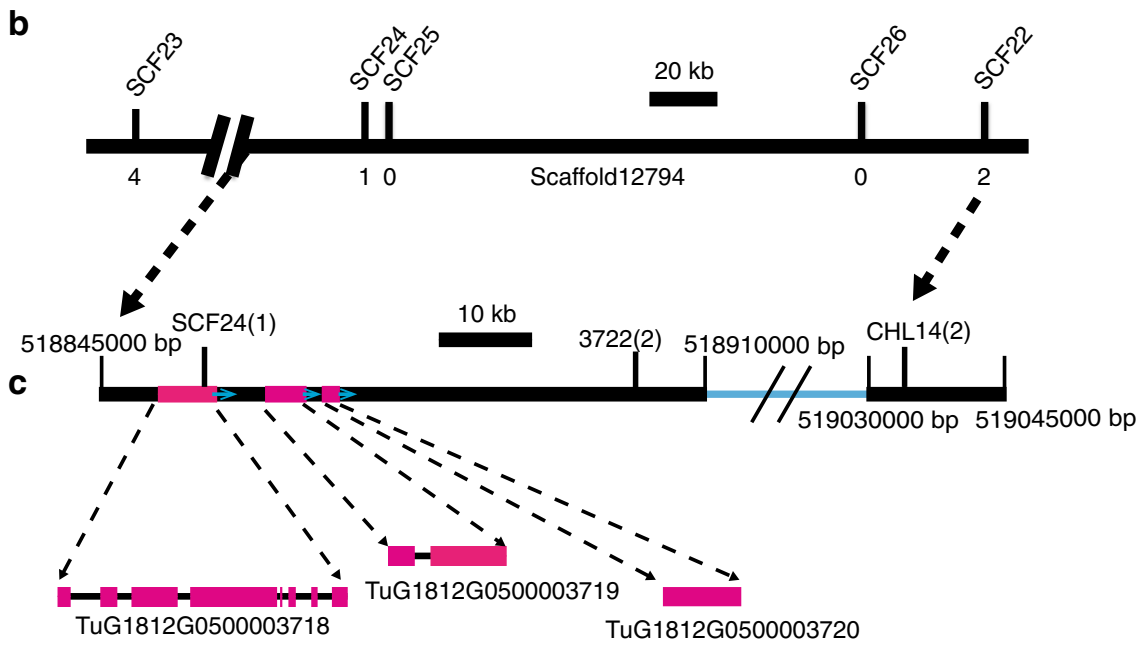

Fig. 2 Genetic and physical maps of the locus conferring resistance to Pst CYR33. a Genetic maps of the locus conferring resistance to Pst CYR33 constructed using $F_{2}$ populations derived from the cross between PI428309 and G1812. b Physical maps of Yr locus on scaffold12794. The number of recombinants are indicated below the scaffold12794. c Physical maps of Yr locus on 518845000 to 519045000 bp of chromosome arm 5AL. The blue line corresponds to 518910000 to 519030000 bp region on the G1812 chromosome arm 5AL. Three genes (TuG1812G0500003718, TuG1812G0500003719 and TuG1812G0500003720) found at the stripe rust resistance (Yr) mapping locus of G1812.

few uredia (Fig. 3). This result indicated that $\mathrm{YrU1}$ is responsible for resistance to Pst CYR33 in PI428309.

Validation of $\mathrm{YrU1}$ gene by transgenic complementation. To further test whether $\mathrm{YrU1}$ is responsible for conferring resistance to stripe rust Pst CYR33, we cloned a genomic fragment of $\mathrm{YrU1}$ from T. urartu PI428309, comprising 2714 bp upstream of the start codon, the coding region and 2086 bp downstream of the stop codon, into the binary vector pCAMBIA-1300. We then introduced the construct into the stripe-rust-susceptible wheat cultivar Bobwhite. We obtained six independent transgenepositive plants, and PCR and GRT-PCR analysis confirmed the presence and expression of the transgene in the $T_{0}, T_{1}$ and $T_{2}$ progeny of three representative transgenic lines (Fig. 4). The infection assays in the $T_{1}$ and $T_{2}$ plants demonstrated that the transgenic plants expressing $\mathrm{YrU1}$ supported only limited uredia and showed prominent cell death at $14 \mathrm{dpi}$, in contrast to Bobwhite and to $\mathrm{T}_{1}$ plants that did not express $\mathrm{YrU1}$, which produced abundant uredia and lacked prominent cell death (Fig. 4b). $\mathrm{T}_{2}$ plants expressing $\mathrm{YrU1}$ also showed resistance to stripe rust races CYR17, CYR32 and V26, and produced fewer uredia than Bobwhite in all cases (Supplementary Fig. 8). Taken together, these results indicate that $\mathrm{YrU} 1$ confers resistance to Pst races CYR33, CYR17, CYR32 and V26 in wheat.

YrU1 encodes an integrated ANK-NLR-WRKY immune receptor. The $Y r U 1$ gene encodes a CC-NBS-LRR protein, with an additional ANK domain at the $\mathrm{N}$ terminus and a WRKY domain at the $\mathrm{C}$ terminus. Unlike previously cloned integrated NLR immune receptors (such as RRS1, RGA5, Yr7, Yr5, YrSP and $\left.\mathrm{Sw}-5 \mathrm{~b}^{7,15,17,21}\right)$, YrU1 has integrated domains at both its $\mathrm{C}$ and $\mathrm{N}$ termini. The domain structure of the $\mathrm{YrU} 1$ protein is very 
a

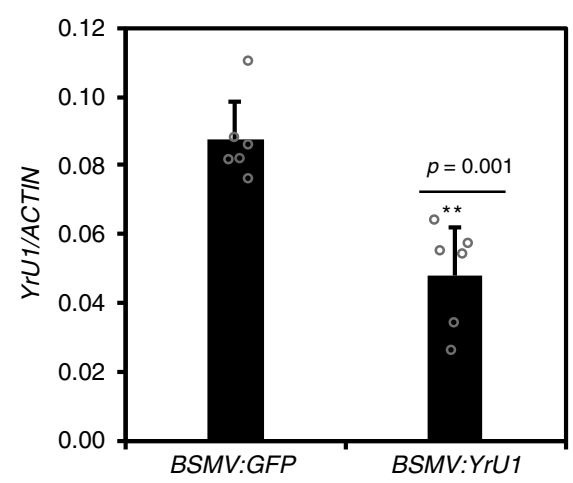

C

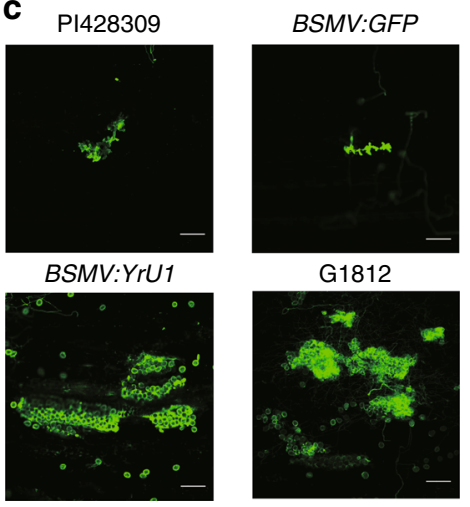

b

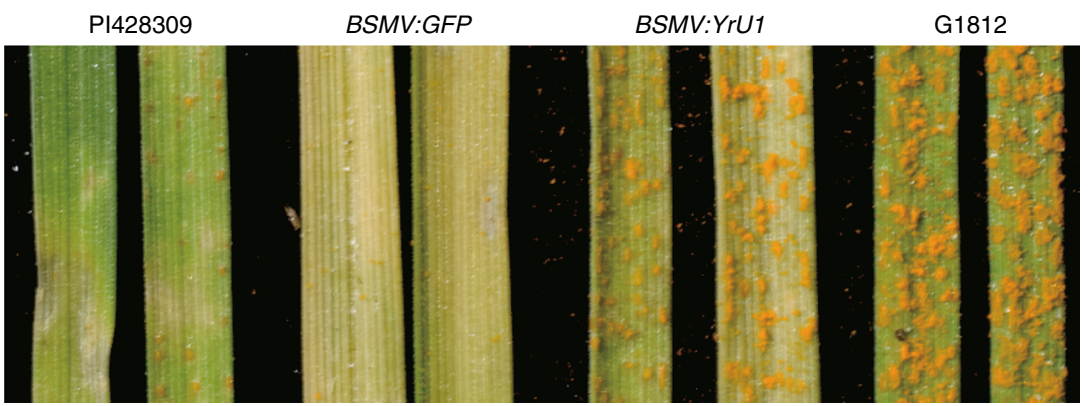

Fig. 3 Barley stripe mosaic virus (BSMV)-induced gene silencing of YrU1 suppresses the resistance of PI428309 to Puccinia striiformis f. sp. tritici (Pst) CYR33. a Relative transcript levels of YrU1 in BSMV:GFP (control) and BSMV:YrU1 plants were examined by qRT-PCR. Results represent the means \pm SD from six independent biological samples. Two asterisks indicate statistically significant difference $(P<0.01$, One-Way ANOVA). b PI428309, BSMV: GFP, BSMV:YrU1 and G1812 plants were inoculated with Pst CYR33 and photographed at $14 \mathrm{dpi}$. c Fluorescence micrographs of the fungal structures of Pst CYR33 at 14 dpi in PI428309, BSMV:GFP, BSMV:YrU1 and G1812 plants. The fungal structures were stained with wheat germ agglutinin (WGA). Bars, $100 \mu \mathrm{m}$. This experiment was repeated at least three times with similar results.

unusual: we identified only three ANK-NLR-WRKY genes across the genomes of all species from the public reference proteome database UniProt. Those three ANK-NLR-WRKY genes are from T. urartu (YrU1), T. aestivum and emmer wheat (Triticum dicoccoides). We also performed a BLASTP analysis with the YrU1 protein in T. durum Svevo whole genome and did not find any ANK-NLR-WRKY protein, indicating that $Y r U 1$-type genes exist only in Triticum species (Fig. 5b, Supplementary Table 5). Sequence alignment indicated that those three proteins have $>90 \%$ sequence identity and the genes are likely to be homologous. There are only two amino acid differences between the two ANK-NLR-WRKY proteins from the wheat cultivar Chinese Spring and the wild emmer accession "Zavitan" (Fig. 5a). The sequences of YrU1 are more divergent from the Chinese Spring and Zavitan proteins than those two are from each other, and the differences between YrU1 and the other two ANK-NLRWRKY proteins exist mainly in the WRKY domains (Fig. 5a). Although Chinese Spring contains an ANK-NLR-WRKY gene that is highly similar to $\mathrm{YrU1}$, the cultivar is susceptible to the Pst CYR33 (Supplementary Fig. 9), indicating that the YrU1 variant in Chinese Spring is not functional in regard to resistance to Pst CYR33.

Beside those three ANK-NLR-WRKY genes, we also identified one ANK-NLR gene (the allelic gene of $Y r U 1$ in G1812) and 17 NLR-WRKY genes across the wheat genomes and the genomes of the related grass species Aegilops tauschii, Triticum urartu, Hordeum vulgare (barley), Brachypodium distachyon, Oryza sativa (rice) and Zea mays (corn) (Fig. 5b, Supplementary Table 5). Sequence alignment of the WRKY domains from the 17 NLR-WRKY proteins indicated that the WRKY heptad domains are relatively conserved (black line in Supplementary Fig. 10). The sequence of this domain of YrU1 is consistent with that of Arabidopsis RRS1. The WRKY heptad domain is detected in acetylated peptides in RRS1, and the $\mathrm{K}$ residues of this domain are necessary for recognition of PopP2 and AvrRps4 ${ }^{20,21}$. Those results suggest that YrU1 may recognise and bind stripe rust effector through the WRKY domain.

We identified 54 additional ANK-domain-containing proteins and 73 WRKY-domain-containing proteins across the T. urartu genome (Supplementary Tables 6 and 7). We constructed a phylogenetic tree based on 56 ANK-containing proteins from $T$. urartu and two ANK-NLR-WRKY proteins from T. aestivum and $T$. dicoccoides, respectively, and a second phylogenetic tree based on 94 WRKY-containing proteins, including 76 from T. urartu, 17 NLR-WRKYs from grasses and RRS1 from Arabidopsis. The dendrogram based on 58 ANK-containing proteins showed that ANK domains are diverse, but are split from ANK-NLR proteins and non-NLR proteins (Fig. 5c). Those results suggest that the integration of ANK domains was most probably derived from one integration event. Similarly, the dendrogram based on 94 WRKYcontaining proteins (except for BGIOSGA022298-PA from rice) show that WRKY domains are diverse, but are split from NLRWRKY proteins and non-NLR proteins (Fig. 5d).

YrU1 overexpression does not induce cell death. To further characterize the function of YrU1, we transiently expressed YrU1 with an N-terminal GFP tag or C-terminal HA tag under the control of the $35 S$ promoter in Nicotiana benthamiana. Overexpression of full-length YrU1 in $N$. benthamiana leaves did not induce cell death. In contrast, the positive control, Pm60 (a protein that conveys resistance to powdery mildew), did induce cell death in N. benthamiana leaves (Supplementary Fig. 11). 
a

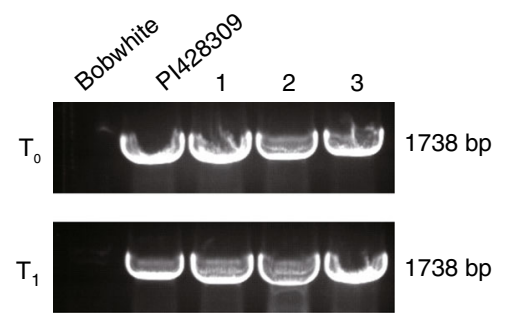

b
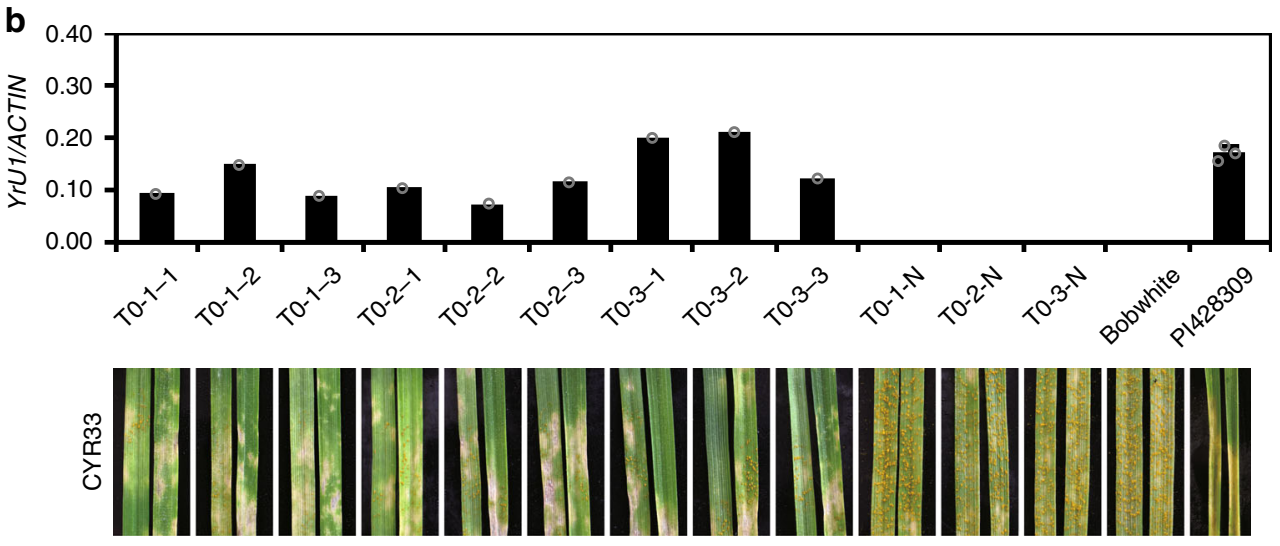

C

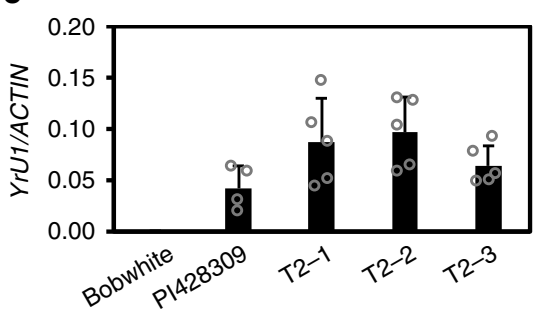

Bobwhite PI428309 T2-1 $\quad$ T2-2 $\quad$ T2-3

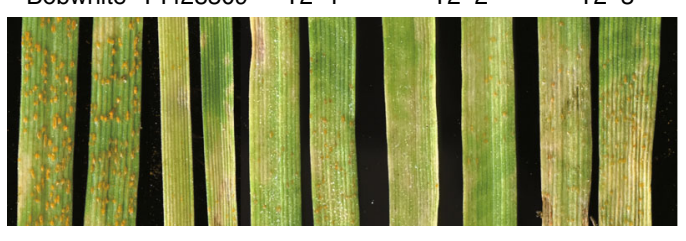

Fig. 4 Validation of YrU1 by transgenic complementation. a Detection of YrU1 gene in susceptible bread wheat cv. Bobwhite and PI428309 and in $T_{0}$ and $\mathrm{T}_{1}$ transgenic plants from three independent lines (Bobwhite background) by PCR. $\mathbf{b}$ Transcript levels of YrU1 as determined by qRT-PCR in $\mathrm{T}_{1}$ transgenic plants from three independent lines (Bobwhite background) and their resistance to Pst CYR33 were evaluated at the seedling stage. T0-1-1, T0-1-2, T0-1-3 and TO-1-N were from the TO-1 line; TO-2-1, TO-2-2, T0-2-3 and TO-2-N were from the TO-2 line; TO-3-1, T0-3-2, TO-3-3 and TO-3-N were from the TO-3 line; and TO-1-N, TO-2-N, TO-3-N and Bobwhite were used as negative controls. PI428309 was used as the positive control. c Transcript levels of YrU1 and resistance to Pst CYR33 in $T_{2}$ plants from three independent lines. Ten-day-old plants were infected with Pst CYR33. The leaves were detached and photographed at $14 \mathrm{dpi}$. Error bars represent the SD from at least three independent biological samples. All experiments were repeated at least three times with similar results.

Next, we overexpressed different domains of YrU1, including the ANK, CC, NB-ARC, LRR and WRKY domains, in N. benthamiana leaves, and found that none of these domains induced cell death (Supplementary Fig. 11). Taken together, those results suggest that YrU1 alone does not induce cell death in N. benthamiana leaves, and cell death may require the presence of pathogen effector or other proteins, similar to what has been reported previously for other NLR proteins with integrated domains, such as RRS1 and RGA5 ${ }^{19,20}$.

YrU1 ANK and CC domains self-associate. Previous studies reported that self-association of the $\mathrm{N}$ terminus of NLR proteins plays a crucial role in triggering downstream immune signals ${ }^{27-29}$. YrU1 contains an N-terminal ANK domain, a type of domain that typically functions in mediating specific protein-protein interactions ${ }^{30}$. This implied that the N-terminal domain of YrU1 might self-associate, as reported previously for other NLR proteins, such as MLA10, Sr33, Sr50, RGA5 and L6 ${ }^{19,27,29}$. To validate this hypothesis, we examined whether N-terminal domains of YrU1 self-associate using yeast two-hybrid, luciferase complementation imaging and co-immunoprecipitation assays (Fig. 6). The results showed that the ANK domain and CC domain of YrU1 can indeed self-associate in vivo and in planta. These results suggest that YrU1 can form N-terminally linked homodimers, a process that might play a role in transducing immune signals.

\section{Discussion}

The wild relatives of wheat are good sources of resistance genes, and many such genes have been cloned, such as Sr33, Pm21 and $\operatorname{Yr} 36^{4,31,32}$. Here, we cloned a stripe rust resistance gene, $\mathrm{YrU1}$, from T. urartu accession PI428309 and determined that it conveys resistance to several races of stripe rust pathogen in common wheat (Supplementary Fig. 8). Therefore, the $\mathrm{YrU1}$ gene is a potentially valuable tool for use in breeding wheat strains for cultivation.

YrU1 is a NLR protein that contains an N-terminal ANK and a C-terminal WRKY domain. ANK-NLR-WRKY proteins are rare: we identified only three ANK-NLR-WRKY proteins from the public reference proteome databases UniProt and Ensemble. All three are found in Triticum species, and their sequences are highly conserved. The common wheat cultivar Chinese Spring, which contains an ANK-NLR-WRKY protein, is susceptible to 
a

YrU1 Triticum urartu \begin{tabular}{|l|l|l|l|l|l|l|}
\hline ANK & & CC & & NB-ARC & & YRK \\
\hline
\end{tabular}

TraesCS5A02G344100 Triticum aestivum

TRIDC5AG050380

Triticum dicoccoides

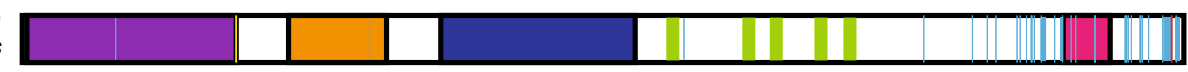

AA polymorphism between TraesCS5A02G344100 and TRIDC5AG050380

AA polymorphism between YrU1 and TraesCS5A02G344100, TRIDC5AG050380

b

\begin{tabular}{l|cccc}
\multicolumn{1}{c|}{ Species } & No & \multicolumn{2}{c}{ No } & No \\
& ANK-NLR-WRKYs & ANK-NLRs & NLR-WRKYs & \\
\hline Triticum urartu & 1 & 1 & 2 & Legend \\
Triticum aestivum & 1 & - & 2 & - NLR-WRKY proteins \\
Triticum dicoccoides & 1 & - & - & - WRKY proteins \\
Aegilops tauschii & - & - & 2 & - WRKY-WRKY proteins \\
Brachypodium distachyon & - & - & 1 & - Non-NLR WRKY-containing proteins \\
Rice & - & - & 9 & - ANK-NLR proteins \\
Hordeum vulgare & - & - & 1 & - Non-NLR ANK-containing proteins \\
Zea mays & - & - & - &
\end{tabular}

C

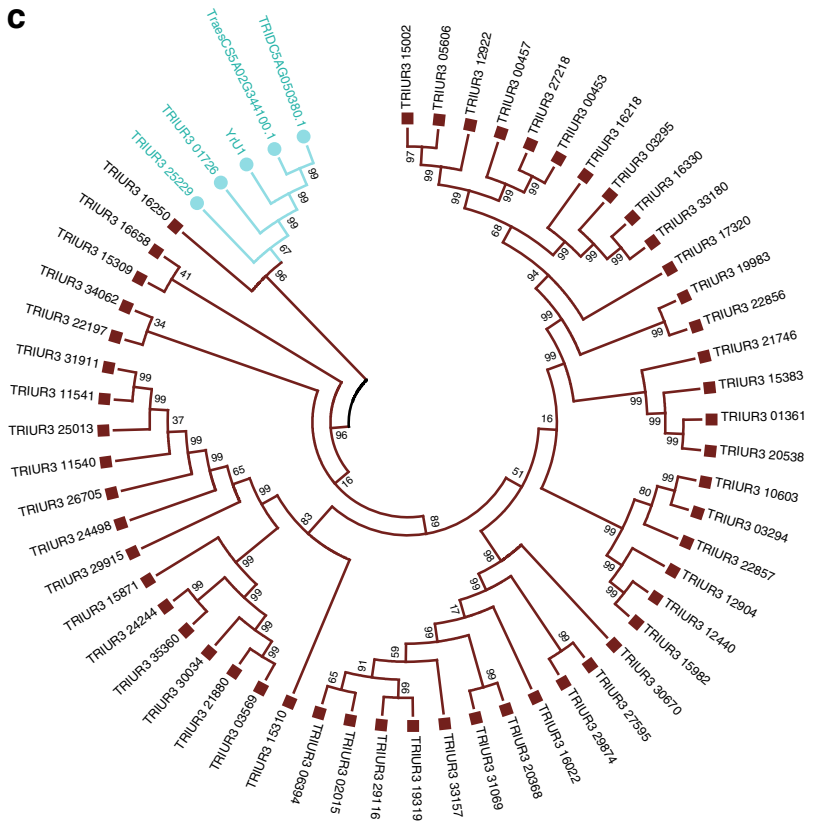

d

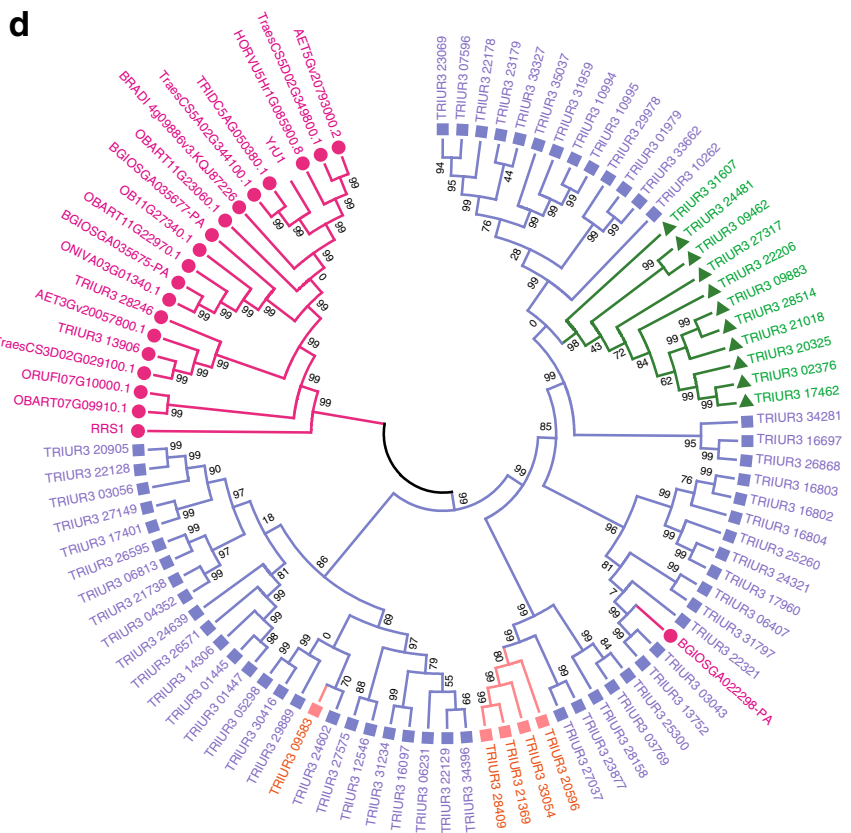

Fig. 5 YrU1 encodes an integrated ANK-NLR-WRKY immune receptor. a Schematic representation of YrU1 protein domain organisation.

TraesCS5A02G344100 and TRIDC5AG050380, from T. aestivum and T. dicoccoides, respectively, also encode ANK-NLR-WRKY proteins. ANK domains are highlighted in purple, CC domains in yellow, NB-ARC domains in blue, LRR motifs in green and WRKY domains in red. Amino acid polymorphisms between YrU1 and the other two ANK-NLR-WRKY proteins (TraesCS5A02G344100 and TRIDC5AG050380) are highlighted with light blue vertical bars, and amino acid polymorphisms of TraesCS5A02G344100 and TRIDC5AG050380 with red vertical bars. Matching colours on the protein structure indicate $100 \%$ sequence conservation. b Numbers of ANK-NLR-WRKY, ANK-NLR and NLR-WRKY genes in different grass genomes (see Supplementary Table 5). c Phylogenetic analysis of 58 ANK-containing proteins including the 56 ANK-containing proteins from T. urartu and two ANK-NLR-WRKY proteins from T. aestivum and T. dicoccoides, respectively. ANK-NLRs are in light blue and non-NLR ANK-containing proteins are in brown. $\mathbf{d}$ Phylogenetic tree analysis with 94 WRKY domains including the 76 WRKY-containing proteins from T. urartu, 17 NLR-WRKYs from grass genomes and RRS1 from Arabidopsis. NLRWRKYs and non-NLR-WRKY-containing proteins are highlighted in red and orange, respectively. WRKY and WRKY-WRKY proteins are in purple and green, respectively. 
a

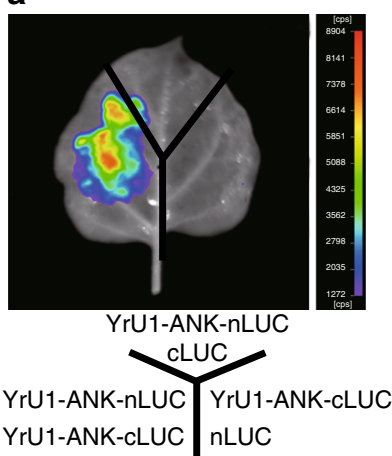

b

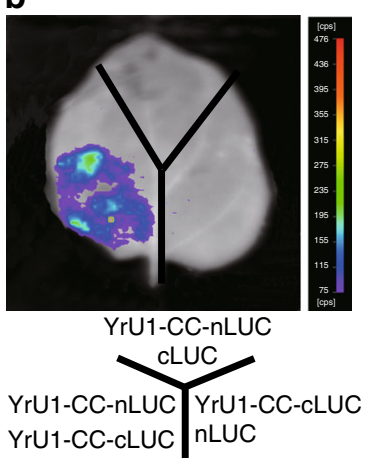

C

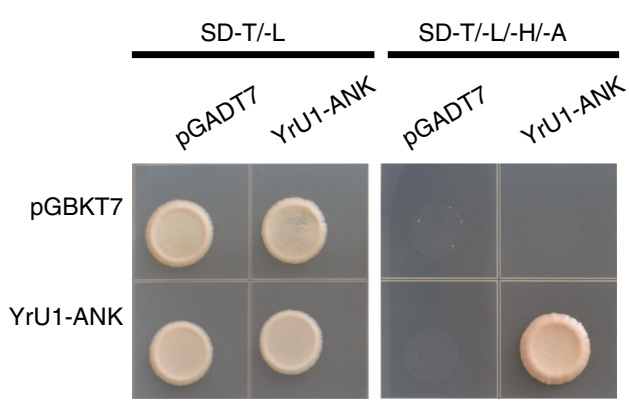

d

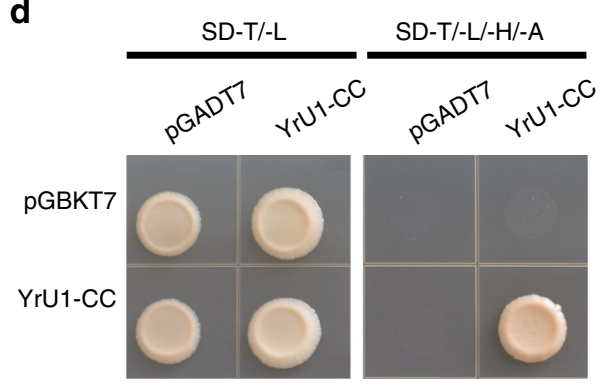

e

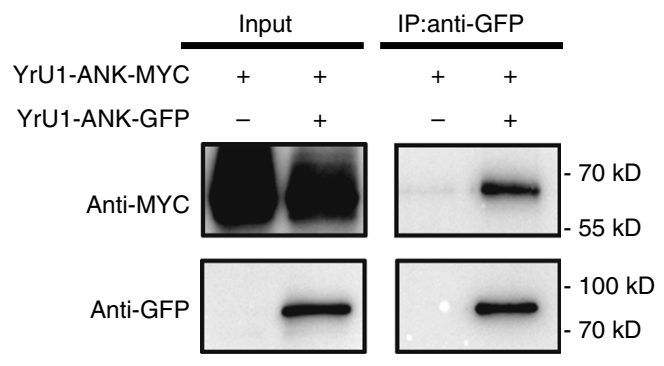

f

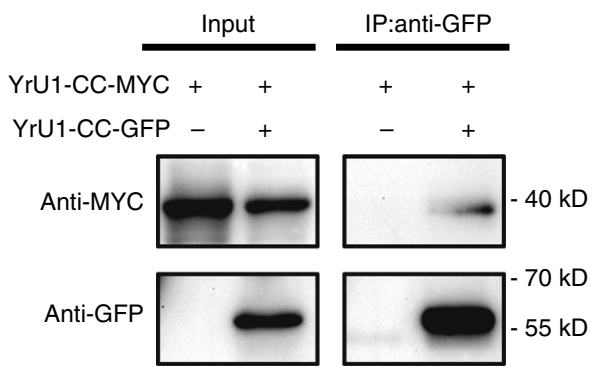

Fig. 6 Self-association of the ANK and CC domains of YrU1. a, b Self-association of ANK (a) and CC (b) domains of YrU1 in firefly luciferase complementation imaging (LUC) assay. c, d Self-association of ANK (c) and CC (d) domains of YrU1 in yeast two-hybrid assays. The coding sequences of YrU1-ANK and YrU1-CC were fused to the Gal4 transactivation domain (AD) or the Gal4 DNA-binding domain (BD). Eight pairs of constructs were cotransformed into $\mathrm{AH} 109$. A $10-\mu \mathrm{L}$ suspension $\left(\mathrm{OD}_{600}=0.5\right)$ of each co-transformant was dropped onto synthetic dropout (SD) medium lacking Leu and Trp and SD medium lacking Ade, His, Leu and Trp. Photographs were taken after $3 \mathrm{~d}$ of incubation. e, $\mathbf{f}$ The ANK (e) and CC (f) domains of YrU1 can selfassociate in planta. Shown are immunoblots with anti-GFP and anti-MYC antibodies of total proteins extracted at $48 \mathrm{~h}$ post inoculation (hpi) from $\mathrm{N}$. benthamiana leaves transiently expressing YrU1-ANK-MYC, YrU1-ANK-GFP, YrU1-CC-MYC or YrU1-CC-GFP and immunoprecipitated with anti-GFP. All experiments were repeated at least two times with similar results.

stripe rust $(P s t)$ race CYR33, indicating that the allelic $Y r U 1$ in Chinese Spring is nonfunctional against Pst CYR33. The high level of identity of the three Triticum ANK-NLR-WRKY proteins suggests that they derive from a common evolutionary origin and that the YrU1 variant in Chinese Spring lost the resistance to stripe rust during the evolution of the rust strains. This type of NLR protein is unique to wheat-related families and may reflect the existence of a specific NLR-mediated disease resistance mechanism in wheat relatives.

Several NLR resistance proteins reported previously have additional domains, such as the Arabidopsis resistance protein RRS1, with a C-terminal WRKY domain; rice blast resistance protein RGA5, with a C-terminal RATX1 domain; tomato resistance protein $\mathrm{Sw}-5 \mathrm{~b}$ has an extra N-terminal domain; and wheat stripe rust resistance proteins Yr5, Yr7 and YrSP, with N-terminal BED domains ${ }^{7,15,18,19}$. YrU1 is the first NLR protein reported with extra domains at both the $\mathrm{N}$ and $\mathrm{C}$ termini. YrU1's $\mathrm{N}$ terminal ankyrin repeats, a 33-amino-acid sequence motif, are generally involved in protein-protein interactions ${ }^{33}$. The bestcharacterized ANK protein from plants is Arabidopsis NPR1, which is a master regulator of the salicylic acid (SA) signalling pathway and functions as a transcriptional coactivator to regulate plant defence response ${ }^{34,35}$. The SUMO-interaction motif (SIM3) located in the ANK domain of NPR1 is modified by SUMO3 to regulate the defence response ${ }^{36}$. Pseudomonas syringae type III effector AvrPtoB targets NPR1 and subverts plant innate immunity by repressing NPR1-dependent SA signalling ${ }^{37}$. ACD6 and BDA1 are also ANK proteins and function as positive regulators of SA signalling in defence responses ${ }^{38-40}$. Although YrU1 contains an ANK domain, it does not show high similarity to NPR1, ACD6 or BDA1. YrU1 is an NLR protein, whose ANK domain may interact with other NLR proteins or act as an integrated decoy domain to interact with the effector of the pathogen, thereby inducing plant immunity.

The C-terminal WRKY domain of YrU1 is a putative transcriptional domain. The Arabidopsis resistance protein RRS1-R is a TIR-NBS-LRR protein with a C-terminal WRKY domain that acts as an integrated decoy to detect and bind the bacterial effectors PopP2 and AvrRps4 to trigger the plant immunity ${ }^{20,21}$. Thus, the YrU1 protein, which has a WRKY domain similar to that of RRS1-R, may recognise and bind stripe rust effector through that domain, thereby activating immune responses.

Here, we showed that the ANK and CC domains of YrU1 selfassociate. However, neither the individual YrU1 domains nor fulllength YrU1 induced cell death in N. benthamiana leaves. NLR oligomerization via $\mathrm{N}$-terminal domains, which are commonly coiled-coil or TIR domains in plants, is thought to initiate disease resistance signals by recruiting or activating downstream signals ${ }^{13,41}$. Indeed, previous studies of several NLR proteins, such as MLA10, Sr33, Sr35, N, L6, RRS1-R/RPS4 and RGA4/RGA5, indicate that their N-terminal domains can selfassociate ${ }^{19,20,27,29,42}$. MLA10, Sr33, Sr35 and L6 can induce an effector-independent cell death response in planta ${ }^{27,29}$. Recent structural work has also shown that oligomerisation is critical for NLR activation ${ }^{43,44}$. However, RRS1-R or RGA5 triggering of cell death requires their partners RPS4 or RGA4 and pathogen 
effectors ${ }^{19,20}$. YrU1, like RPS4 or RGA4, may require other NLR proteins and pathogen to trigger the defence responses.

It is possible that YrU1, like previously identified NLR proteins (such as RRS1 and RGA5), initiates the resistance response by recognising and binding a stripe rust effector through integrated decoy domains. However, YrU1 differs from these other NLR proteins in having integrated domains at both its $\mathrm{C}$ and $\mathrm{N}$ termini, and YrU1-type proteins exists only in wheat and its relatives. These two domains may thus play different roles in activating resistance responses than other NLR proteins do, and YrU1-mediated disease resistance may represent a mechanism present only in wheat. Activation of YrU1 may represent a unique molecular mechanism in NLR-mediated plant immunity. Based on current information, we present a working model of how YrU1 functions (Supplementary Fig. 12). In this model, YrU1 is in the rest state in the absence of pathogen. When stripe rust pathogen is present, the effector binds to the WRKY domain, resulting in conformational changes and oligomerisation of $\mathrm{YrU} 1$, which leads to activation of disease resistance. The ANK domain may recruit additional components to activate downstream signalling, or it may also act as a decoy for effector binding. It would be very interesting to determine the roles of the integrated ANK and WRKY domains in perceiving and transducing the immune signals mediated by YrU1. Identification of corresponding effector(s) recognised by YrU1 would be the key to understanding YrU1 molecular mechanism.

\section{Methods}

Plant materials. Triticum urartu accession PI427328 was collected from Shaqlawa, Iraq. The PI428209, PI428214, PI428294 and G1812 accessions were collected from Mardin, Turkey. The whole-genome shotgun draft sequence of the G1812 accession has been published ${ }^{24}$. The PI428309 accession was collected from El Beqaa, Lebanon. No stripe rust resistance genes were reported in these accessions. The high-density genetic map was generated from the $\mathrm{F}_{2}$ population derived from a cross between resistant T. urartu accession PI428309 and susceptible T. urartu accession G1812. The hexaploid winter wheat cultivar mingxian169 (susceptible to stripe rust races CYR17, CYR31, CYR32, CYR33 and V26) was used to maintain the Pst race used in the experiments. The susceptible spring common wheat cultivar Bobwhite was used to generate the transgenic plants.

Stripe rust assays. Pst races CYR17, CYR31, CYR32, CYR33 and V26 were used in the experiments. We inoculated the stripe rust by spraying the mixture of urediospores and talcum powder at a ratio of 1:2 at the two-leaf stage, and talcum powder was used as an indicator of the urediospores on the leaves during the inoculation ${ }^{9}$. Immediately after inoculation, plants were incubated in a greenhouse at $10^{\circ} \mathrm{C}$ with $100 \%$ relative humidity in the dark for $24 \mathrm{~h}$. The plants were then moved to a greenhouse at $16-18^{\circ} \mathrm{C}$ under a 14 -h-light/10-h-dark cycle. The disease infection type (IT) was evaluated at $14 \mathrm{dpi}$. Stripe rust infection types were assessed based on a 0-4 scale ${ }^{45}$. In details, IT 0: immune, no visible uredia and necrosis on leaves; IT: nearly immune, no uredia with hypersensitive flecks on leaves; IT 1: very resistant, few small uredia with distinct necrosis on leaves; IT 2: moderately resistant, few small- to medium sized uredia with dead or chlorosis on leaves; IT 3: moderately susceptible, a lot of medium-sized uredia, no necrosis, but with chlorosis on leaves; IT 4: highest susceptible, a large number of large-sized uredia without necrosis on leaves.

Microscopy. To visualise the average infection area, the infected leaves were detached at 2 and 14 dpi and stained with WGA-FITC (L4895-10MG; Sigma) as described previously ${ }^{46,47}$, with minor modification. Briefly, the leaves were cut into $2-\mathrm{cm}$ pieces and placed in a $10 \mathrm{ml}$ centrifuge tube with $5 \mathrm{ml}$ of $1 \mathrm{M} \mathrm{KOH}$ and $0.05 \%$ Silwet L-77. After $12 \mathrm{~h}$, the $\mathrm{KOH}$ solution was gently poured off and washed with $10 \mathrm{ml}$ of $50 \mathrm{mM}$ Tris ( $\mathrm{pH} 7.5$ ). This solution was then replaced with another $10 \mathrm{ml}$ of $50 \mathrm{mM}$ Tris (pH 7.5). After $20 \mathrm{~min}$, the Tris solution was removed and replaced with $5 \mathrm{ml}$ of $20 \mathrm{\mu g} \mathrm{ml}^{-1}$ WGA-FITC. Tissue was stained for $15 \mathrm{~min}$ and then washed with $50 \mathrm{mM}$ Tris (pH 7.5). The WGA-FITC-stained tissue was examined under blue light excitation with a Zeiss LSM 880 confocal microscope.

Development of molecular markers. The simple sequence repeat (SSR) markers on hexaploid wheat chromosomes (1A to $7 \mathrm{~A}$ ) were used to generate the initial genetic map of $\mathrm{YrU1}$. First, the SSR markers were used to screen the polymorphisms between PI428309 and G1812, and then eighty-two polymorphic markers were used to construct linkage map using the susceptible plants of the $F_{2}$ population derived from the cross between PI428309 and G1812. The genetic map was constructed by software JoinMap 4.0. Closely linked flanking markers were developed from the scaffolds of G1812 and designed using SSR Locator software ${ }^{48}$ and dCAPS Finder 2.0 (http://helix.wustl.edu/dcaps/dcaps.html). The polymorphic markers were used to screen the susceptible plants of the $F_{2}$ population for the recombinants. In total, 3304 susceptible plants were used to map the resistance gene.

Quantitative real-time RT-PCR. Total RNA was isolated using TRIzol reagent (Invitrogen). First-strand cDNA from total RNA was synthesized using murine leukemia virus reverse transcriptase (Promega) and quantitative reverse transcription PCR (qRT-PCR) was performed with SYBR green kit (Takara) following the manufacturer's instructions.

Cloning of the stripe rust resistance gene. In order to clone the candidate genes in PI428309, we designed primers based on the sequences of TuG1812G0500003718, TuG1812G0500003719 and TuG1812G0500003720. We obtained the DNA sequences of two candidate genes in PI428309, which we designated $\mathrm{YrU1}$ and $\mathrm{CG} 2$ based on subsequent analysis, by PCR amplification using gene specific primers YrU1-C and CG2-C according to the sequences of TuG1812G0500003718 and TuG1812G0500003719, respectively. We obtained the coding sequence of YrU1 by PCR amplification from the cDNA of PI428309 using the primer YrU1-C, however, we could not obtain the coding sequence of CG2 as expression of CG2 was not detected. We also validated the coding sequence of YrU1 by rapid amplification of 3' and 5' cDNA ends.

Prediction and annotation of coding sequences of $\boldsymbol{Y r U 1}$. The predicted open reading frame of the coding sequence based on the sequence of 3' and 5' RACE was performed with the online programs (http://www.softberry.com/berry.phtml? topic $=$ fgenesh\&group $=$ programs\&subgroup $=$ gfind) and translated using the DNAMAN tool (v9.0.1.116). The YrU1 encoded an ANK-NLR-WRKY protein based on the functional annotation using the SMART program (http://smart.emblheidelberg.de/), the LRRsearch program (http://lrrsearch.com/) and the CD-search online tool (https://www.ncbi.nlm.nih.gov/Structure/cdd/wrpsb.cgi).

Wheat transformation. The 11,858-bp genomic fragment of $\mathrm{YrU} 1$, including the $2714 \mathrm{bp}$ upstream of the start codon, all the exons and introns and $2086 \mathrm{bp}$ downstream of the stop codon, was cloned into the vector pCAMBIA-1300. The YrU1 genomic fragment in the pCAMBIA-1300 vector was introduced into the stripe-rust-susceptible wheat cultivar Bobwhite by particle bombardment as described previously 26,49

Phylogenetic analyses. We used the neighbour-joining method implemented in MEGA (v7.0.26) to analyse the relationships between ANK domains from ANKNLR and non-NLR proteins. The phylogenetic tree analyses were performed as described previously ${ }^{7}$, with a little alteration. First, we retrieved all ANK-containing proteins from the T. urartu genome and ANK-NLR proteins from grass genomes using the following steps: we used hmmer (v3.1b2, http://hmmer.org/) (UniProt references proteomes) to identify conserved domains in protein sequences from the T. urartu genome. We applied default parameters to filter out any unrelated identified domains. ANK domains of the corresponding proteins in the output from hmmer were verified on the $\mathrm{CD}$-search database. We divided the group between ANK-NLRs and non-NLRs based on the presence of NB-ARC domains. The phylogenetic tree analyses were performed in MEGA (v7.0.26). We used the same method to generate a phylogenetic tree of WRKY domains.

Yeast two-hybrid assays. Yeast two-hybrid assays were performed in the Matchmaker GAL4 Two-Hybrid System 3 (Clontech). To examine the selfassociation of the ANK and CC domains of YrU1, the coding sequences of YrU1ANK and YrU1-CC were cloned into pGADT7 or pGBKT7. Different pairs of constructs were co-transformed into Saccharomyces cerevisiae strain AH109.

Immunoblot analysis. Different plasmids transformed into Agrobacterium tumefaciens GV3101 were suspended in infiltration buffer as previously described ${ }^{50}$ to $\mathrm{OD}_{600}=1.5$. Transient expression, total protein extraction and immunoblot analysis were performed as described ${ }^{51}$, with minor alterations. Total protein extracts $(1 \mathrm{ml})$ were incubated with $20 \mu \mathrm{L}$ agarose-conjugated anti-GFP antibody (MBL, D153-8) at $4{ }^{\circ} \mathrm{C}$ for $4 \mathrm{~h}$ with gentle rotation. After incubation, the agarose beads were washed four times with phosphate-buffered saline (PBS) containing 0.008$0.01 \%(\mathrm{v} / \mathrm{v})$ Triton X-100 and resuspended in $80 \mu \mathrm{L}$ PBS; $20 \mu \mathrm{L} 5 \times$ SDS/PAGE loading buffer was added and then the mixture was boiled for $5 \mathrm{~min}$. The proteins were detected with anti-MYC (Abmart, M2002), anti-GFP (Abmart, M2004) and anti-HA (Abmart, M2003) immunoblot.

Luciferase complementation imaging assay. To examine the self-association of the ANK and CC domains of YrU1, the coding sequences of YrU1-ANK and YrU1-CC were cloned into pCAMBIA1300-nLUC or pCAMBIA1300-cLUC. YrU1-ANK-nLUC/YrU1-ANK-cLUC or YrU1-CC-nLUC/YrU1-CC-cLUC were 
cotransformed into N. benthamiana by infiltration with Agrobacterium GV3101 After 2 days, $1 \mathrm{mM}$ precooled luciferin was sprayed onto the leaves, and the samples were incubated in the dark for 5-10 min. LUC images were captured using a cooled CCD imaging apparatus ${ }^{52}$.

Oligonucleotide sequences. Primers used in this study are listed in Supplementary Table 8.

Reporting summary. Further information on research design is available in the Nature Research Reporting Summary linked to this article.

\section{Data availability}

Data that supporting the findings of this study are presented in the manuscript and the Supplementary files. Sequence data of $Y r U 1$ gene in PI428309 can be found in the GenBank database under the accession number MT018453. Source data of Figs. 1, 3, 4 and 6, as well as Supplementary Figs. 1-4, 7-9 and 11 are provided as a Source Data file.

Received: 7 November 2019; Accepted: 18 February 2020;

Published online: 13 March 2020

\section{References}

1. Chen, W., Wellings, C., Chen, X., Kang, Z. \& Liu, T. Wheat stripe (yellow) rust caused by Puccinia striiformis f. sp. tritici. Mol. Plant Pathol. 15, 433-446 (2014).

2. Beddow, J. M. et al. Research investment implications of shifts in the global geography of wheat stripe rust. Nat. Plants 1, 15132 (2015).

3. Pakeerathan, K. et al. Identification of a new source of stripe rust resistance Yr82 in wheat. Theor. Appl. Genet. 132, 3169-3176 (2019).

4. Fu, D. et al. A kinase-START gene confers temperature-dependent resistance to wheat stripe rust. Science 323, 1357-1360 (2009).

5. Krattinger, S. G. et al. A putative $\mathrm{ABC}$ transporter confers durable resistance to multiple fungal pathogens in wheat. Science 323, 1360-1363 (2009).

6. Moore, J. W. et al. A recently evolved hexose transporter variant confers resistance to multiple pathogens in wheat. Nat. Genet. 47, 1494-1498 (2015).

7. Marchal, C. et al. BED-domain-containing immune receptors confer diverse resistance spectra to yellow rust. Nat. Plants 4, 662-668 (2018).

8. Klymiuk, V. et al. Cloning of the wheat Yr15 resistance gene sheds light on the plant tandem kinase-pseudokinase family. Nat. Commun. 9, 3735 (2018).

9. Zhang, C. et al. An ancestral NB-LRR with duplicated 3'UTRs confers stripe rust resistance in wheat and barley. Nat. Commun. 10, 4023 (2019).

10. Hovmoller, M. S., Sorensen, C. K., Walter, S. \& Justesen, A. F. Diversity of Puccinia striformis on cereals and grasses. Annu. Rev. Phytopathol. 49, 197-217 (2011).

11. Schwessinger, B. Fundamental wheat stripe rust research in the 21st century. New. Phytol. 213, 1625-1631 (2016).

12. Wang, Z. et al. Virulence variations of Puccinia striiformis $\mathrm{f}$. sp. tritici Isolates collected from Berberis spp. in China. Plant Dis. 100, 131-138 (2016).

13. Jones, J. D., Vance, R. E. \& Dangl, J. L. Intracellular innate immune surveillance devices in plants and animals. Science 354, aaf6395 (2016).

14. Li, X., Kapos, P. \& Zhang, Y. NLRs in plants. Curr. Opin. Immunol. 32, 114-121 (2015).

15. Chen, X. et al. A multilayered regulatory mechanism for the autoinhibition and activation of a plant CC-NB-LRR resistance protein with an extra Nterminal domain. New. Phytol. 212, 161-175 (2016).

16. Mucyn, T. S. et al. The tomato NB-ARC-LRR protein Prf interacts with Pto kinase in vivo to regulate specific plant immunity. Plant Cell 18, 2792-2806 (2006).

17. Cesari, S. et al. The rice resistance protein pair RGA4/RGA5 recognizes the Magnaporthe oryzae effectors AVR-Pia and AVR1-CO39 by direct binding. Plant Cell 25, 1463-1481 (2013).

18. Deslandes, L. et al. Resistance to Ralstonia solanacearum in Arabidopsis thaliana is conferred by the recessive RRS1-R gene, a member of a novel family of resistance genes. Proc. Natl Acad. Sci. USA 99, 2404-2409 (2002).

19. Cesari, S. et al. The NB-LRR proteins RGA4 and RGA5 interact functionally and physically to confer disease resistance. EMBO J. 33, 1941-1959 (2014).

20. Sarris, P. F. et al. A plant immune receptor detects pathogen effectors that target WRKY transcription factors. Cell 161, 1089-1100 (2015).

21. Le Roux, C. et al. A receptor pair with an integrated decoy converts pathogen disabling of transcription factors to immunity. Cell 161, 1074-1088 (2015).

22. Dvorák, J., Terlizzi, P., Zhang, H. B. \& Resta, P. The evolution of polyploid wheats: identification of the A genome donor species. Genome 36, 21-31 (1993).
23. Peng, J. H., Sun, D. \& Nevo, E. Domestication evolution, genetics and genomics in wheat. Mol. Breed. 28, 281-301 (2011).

24. Ling, H. Q. et al. Draft genome of the wheat A-genome progenitor Triticum urartu. Nature 496, 87-90 (2013).

25. Ling, H. Q. et al. Genome sequence of the progenitor of wheat A subgenome Triticum urartu. Nature 557, 424-428 (2018).

26. Zou, S., Wang, H., Li, Y., Kong, Z. \& Tang, D. The NB-LRR gene Pm60 confers powdery mildew resistance in wheat. New. Phytol. 218, 298-309 (2018).

27. Cesari, S. et al. Cytosolic activation of cell death and stem rust resistance by cereal MLA-family CC-NLR proteins. Proc. Natl Acad. Sci. USA 113, 10204-10209 (2016).

28. Schreiber, K. J., Bentham, A., Williams, S. J., Kobe, B. \& Staskawicz, B. J. Multiple domain associations within the Arabidopsis immune receptor RPP1 regulate the activation of programmed cell death. PLoS Path. 12, e1005769 (2016).

29. Bernoux, M. et al. Structural and functional analysis of a plant resistance protein TIR domain reveals interfaces for self-association, signaling, and autoregulation. Cell Host Microbe 9, 200-211 (2011).

30. Mosavi, L. K., Cammett, T. J., Desrosiers, D. C. \& Peng, Z. Y. The ankyrin repeat as molecular architecture for protein recognition. Protein Sci. 13, 1435-1448 (2004).

31. Periyannan, S. et al. The gene Sr33, an Ortholog of barley Mla genes, encodes resistance to wheat stem rust race Ug99. Science 341, 786-788 (2013).

32. Cao, A. et al. Serine/threonine kinase gene Stpk-V, a key member of powdery mildew resistance gene $P m 21$, confers powdery mildew resistance in wheat. Proc. Natl Acad. Sci. USA 108, 7727-7732 (2011).

33. Sedgwick, S. G. \& Smerdon, S. J. The ankyrin repeat: a diversity of interactions on a common structural framework. Trends Biochem. Sci. 24, 311-316 (1999).

34. Mou, Z., Fan, W. \& Dong, X. Inducers of plant systemic acquired resistance regulate NPR1 function through redox changes. Cell 113, 935-944 (2003).

35. Spoel, S. H. et al. Proteasome-mediated turnover of the transcription coactivator NPR1 plays dual roles in regulating plant immunity. Cell 137, 860-872 (2009).

36. Saleh, A. et al. Posttranslational modifications of the master transcriptional regulator NPR1 enable dynamic but tight control of plant immune responses. Cell Host Microbe 18, 169-182 (2015).

37. Chen, H. et al. A bacterial type III effector targets the master regulator of salicylic acid signaling, NPR1, to subvert plant immunity. Cell Host Microbe 22, 777-788.e7 (2017)

38. Lu, H., Rate, D. N., Song, J. T. \& Greenberg, J. T. ACD6, a novel ankyrin protein, is a regulator and an effector of salicylic acid signaling in the Arabidopsis defense response. Plant Cell 15, 2408-2420 (2003).

39. Yang, Y. et al. The ankyrin-repeat transmembrane protein BDA1 functions downstream of the receptor-like protein SNC2 to regulate plant immunity. Plant Physiol. 159, 1857-1865 (2012).

40. Todesco, M. et al. Activation of the Arabidopsis thaliana immune system by combinations of common ACD6 alleles. PLoS Genet. 10, e1004459 (2014).

41. Wang, W., Feng, B., Zhou, J. M. \& Tang, D. Plant immune signaling: advancing on two frontiers. J. Integr. Plant Biol. 62, 2-24 (2020).

42. Mestre, P. \& Baulcombe, D. C. Elicitor-mediated oligomerization of the tobacco N disease resistance protein. Plant Cell 18, 491-501 (2006).

43. Wang, J. et al. Ligand-triggered allosteric ADP release primes a plant NLR complex. Science 364, eaav5868 (2019).

44. Wang, J. et al. Reconstitution and structure of a plant NLR resistosome conferring immunity. Science 364, eaav5870 (2019).

45. Z., K., lili, H. \& H., B. Ultrastructural changes and localization of lignin and callose in compatible and incompatible interactions between wheat and Puccinia striiformis. J. Plant Dis. Prot. 109, 25-37 (2002).

46. Zhang, W. et al. Identification and characterization of Sr13, a tetraploid wheat gene that confers resistance to the Ug99 stem rust race group. Proc. Natl Acad. Sci. USA 114, E9483-E9492 (2017).

47. Ayliffe, M. et al. Nonhost Resistance of Rice to Rust. Pathog. Mol. PlantMicrobe . 24, 1143-1155 (2011).

48. Maia, L. C. D. et al. SSR locator: tool for simple sequence repeat discovery integrated with primer design and PCR simulation. Int. J. Plant Gen. 2008, 412696 (2008).

49. Wang, Y. et al. Simultaneous editing of three homoeoalleles in hexaploid bread wheat confers heritable resistance to powdery mildew. Nat. Biotechnol. 32, 947 (2014).

50. Liu, L. et al. An efficient system to detect protein ubiquitination by agroinfiltration in Nicotiana benthamiana. Plant J. 61, 893-903 (2010).

51. Zhao, Y., Wu, G., Shi, H. \& Tang, D. RECEPTOR-LIKE KINASE 902 Associates with and phosphorylates BRASSINOSTEROID-SIGNALING KINASE1 to regulate plant immunity. Mol. Plant 12, 59-70 (2019).

52. Chen, H. et al. Firefly luciferase complementation imaging assay for proteinprotein interactions in plants. Plant Physiol. 146, 368-376 (2008). 


\section{Acknowledgements}

We thank United States Department of Agriculture (USDA) for providing us with T. urartu material. This work was supported by grants from the National Natural Science Foundation of China (31830077) and National Science Fund for Distinguished Young Scholars of China (31525019) to D.T.

\section{Author contributions}

H.W. and D.T. conceived the research; H.W. and S.Z. performed the experiments; H.W. and D.T. analysed the data; Y.L. contributed Triticum urartu lines; F.L. performed the rust inoculation; H.W. and D.T. wrote the paper; and all authors contributed to revision of the paper.

\section{Competing interests}

We have filed a patent application based on this work. The patent applicant is Fujian Agriculture and Forestry University and the inventors are Dingzhong Tang, Huan Wang and Shenghao Zou. The application number is 202010072144.3. The patent application has been submitted for consideration, and the cloning and validation of $\mathrm{YrU} 1$ described in this paper are covered in the patent application. Other authors are not listed in the patent application and declare no competing interests.

\section{Additional information}

Supplementary information is available for this paper at https://doi.org/10.1038/s41467020-15139-6.

Correspondence and requests for materials should be addressed to D.T.
Peer review information Nature Communications thanks the anonymous reviewer(s) for their contribution to the peer review of this work. Peer reviewer reports are available.

Reprints and permission information is available at http://www.nature.com/reprints Publisher's note Springer Nature remains neutral with regard to jurisdictional claims in published maps and institutional affiliations.

\section{(c) (i)}

Open Access This article is licensed under a Creative Commons Attribution 4.0 International License, which permits use, sharing, adaptation, distribution and reproduction in any medium or format, as long as you give appropriate credit to the original author(s) and the source, provide a link to the Creative Commons license, and indicate if changes were made. The images or other third party material in this article are included in the article's Creative Commons license, unless indicated otherwise in a credit line to the material. If material is not included in the article's Creative Commons license and your intended use is not permitted by statutory regulation or exceeds the permitted use, you will need to obtain permission directly from the copyright holder. To view a copy of this license, visit http://creativecommons.org/ licenses/by/4.0/.

(c) The Author(s) 2020 